\title{
Commentary
}

\section{A novel and accurate technique of photographic wound measurement}

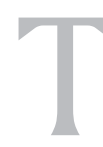

he authors have to be congratulated for describing a relatively new method for measuring the size of the wounds which is in line with the current buzzword, i.e., practicing EBM or 'Evidence-based Medicine'.

Most patients, the treating surgeons, as well as their referring physicians would be interested in evaluating the progress of the patient's condition. In the current scenario, the wound size is not only a guide to the adequacy of the therapeutic measures on offer but also helps to assess the need for a change, if any. But we need to balance the effort required against cheaper and simpler methods. If the authors mention the average time spent per patient for the calculations, it would help others to decide.

Photographs are time tested and - to use a less used, but more appropriate term - routinized. The authors state that the direction, distance and angle of the camera are obviated through the software. Even if correct, such inaccuracies are even better obviated by the discerning eye of a surgeon. Question is, does the extra effort and time spent on the computer score over a simple series of photographs? However, it definitely would be helpful for research purposes.

Similarly, the quote "You can't manage what you can't measure" seems to miss the fact that any brain, let alone

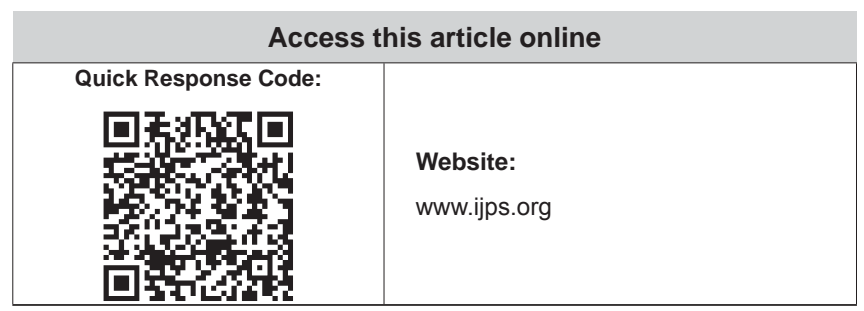

that of an experienced clinician, can calculate much better than any software. While agreeably, such calculations can never be mathematically quantified for publication, a busy clinician, who likes to take decisions in a snap and move on to the next patient, would in all likelihood give such a method a miss.

The authors have innovated in providing a specially created $4 \times 4$-cm label stuck in an adjoining area. Wonder why a simple commercially available graph paper could not have done the trick. In fact, a transparent graph paper stuck right on the wound, would remove even the few relative inaccuracies in the measurement - as the curve in the lower third of the leg is far more than in the middle or upper third. It would also be more time saving. Automation then would be possible and the method advocated could possibly be routinized!

In summary, do try if you are doing cutting edge research on wound care, with a warning that publishing a series of photographs might be adequate too.

PS:- Image J can be freely downloaded from http://rsb.info. nih.gov/ij/download.html. Like all software, there is some learning involved.

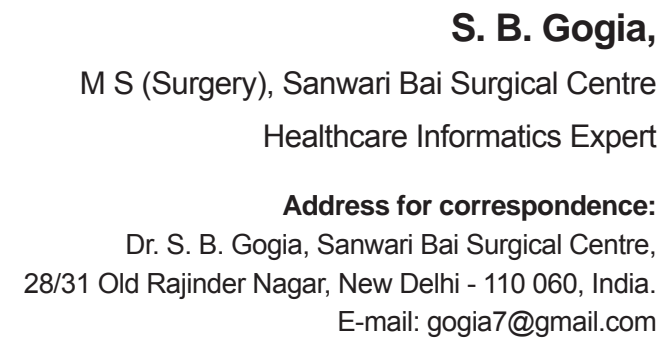

Indian Journal of Plastic Surgery May-August 2012 Vol 45 Issue 2 